\title{
Effects of anisotropic diffraction on micro-periodic structure composed of polymer and liquid crystal phases
}

\author{
Akifumi Ogiwara ${ }^{1 *}$, and Hiroshi Kakiuchida ${ }^{2}$ \\ 1 Department of Electronic Engineering, Kobe City College of Technology, 8-3 Gakuen-higashi, Nishiku, Kobe 651-2194, Japan \\ 2 Materials Research Institute for Sustainable Development, National Institute of Advanced Industrial Science and Technology \\ (AIST), 2266-98 Anagahora, Shimoshidami, Moriyama, Nagoya, 463-8560, Japan \\ * Akifumi Ogiwara: e-mail: ogiwara@kobe-kosen.ac.jp
}

\begin{abstract}
A micro-periodic structure composed of polymer and liquid crystal (LC) phases is called holographic polymer dispersed liquid crystal (HPDLC) grating. The HPDLC grating has been applied for grating formation to obtain both high optical efficiency and electric switching function. Moreover, HPDLC grating shows polarization dependence in diffraction efficiencies against the incident polarization state of laser light. The anisotropic diffraction is modulated by the distribution of submicrometer-scale LC droplet in a polymer matrix using interferometric laser exposure. The refractive-index modulations in grating medium corresponding to the three dimensional directions are obtained by applying theoretical calculation based on the anisotropy for the experimental results of angular dependence of diffraction efficiencies in various grating structures. The anisotropy in grating medium is analyzed by connecting the experimental results of angular dependence of diffraction efficiencies with SEM observations in different grating spacing.

Key words: polymer, liquid crystal, anisotropy, diffraction
\end{abstract}

\section{INTRODUCTION}

The development of holographic and diffractive optical technologies is of considerable interest for integrated optics, sensor systems, displays, optical interconnects, and optical data storage devices. In many applications, it is desirable to have control over the diffraction efficiency of a device by applying electric field. A useful approach is to employ a liquid-crystal (LC) phase, which can consider the change in birefringence induced by electric field as refractive index modulation in the device. Such a device has been realized using a polymer-dispersed liquid crystal (PDLC) consisting of LC droplets embedded in a polymer matrix. The refractive index modulation in PDLC systems is realized by spatial density or concentration variation in the resulting polymer, or spatially distributed voids. Thus, when a mixture of LC and monomer materials containing a photo initiator is irradiated using a spatially periodic intensity, the formation of polymer networks is induced in the bright regions of fringes. At the same time as the reaction, LC molecules are separated in the dark regions of fringes. As a result, a periodic structure consisting of polymerized and LC concentration regions are produced in the PDLC systems using the obtained irradiation pattern. If a holographic optical system with a laser source is applied to the PDLC systems, fine periodic structures produced by cured-polymer and separated LC phases, that is, a holographic polymer-dispersed liquid crystal (HPDLC) grating, can be realized under the control of optical system [1-8].

Diffusion and phase-separation processes during grating formation, which are induced by photo polymerization, are considered to dominate the diffraction properties of the fabricated HPDLC gratings. The high polarization dependence of the diffraction property developed in the HPDLC gratings is expected to provide novel polarization-controllable optical devices, such as thin polarized beam splitting films and anisotropic holographic memory devices [9-12]. Therefore, the anisotropic diffraction properties in the HPDLC gratings are theoretically and experimentally important issues to be studied for the development of novel optical devices. We report in this article the anisotropic diffraction of HPDLC gratings based on the refractive-index modulations in the different grating structure.

\section{THEORETICAL TREATMENT}

Figure 1 shows the model of a thick grating structure for theoretical treatment. The amplitudes of refractive-index modulation $\left(\mathrm{n}_{\mathrm{x}}, \mathrm{n}_{\mathrm{y}}, \mathrm{n}_{\mathrm{z}}\right)$ in grating medium corresponding to the three dimensional directions are estimated by expanding the Kogelnik's coupled-wave theory using the incident angle dependence in anisotropic diffraction against the $\mathrm{p}$ - and s- polarizations $[6,13]$. The estimation procedure is described as follows. The difference in the amplitude of refractive-index modulation in the grating between $\mathrm{p}$ and s-polarizations is regarded as an indicator of the order of LC orientation. The amplitude of refractive-index modulation in the grating is related to diffraction efficiency $(\eta)$ for incident light angle $\left(\theta_{\rho}\right)$ in grating medium through Kogelnik's coupled-wave theory as expressed by [13] 


$$
\eta\left(\theta_{\rho}\right)=\frac{\sin ^{2} \sqrt{v^{2}+\xi^{2}}}{1+\frac{\xi^{2}}{v^{2}}}
$$

where $v=\kappa d\left(\kappa_{p}\right.$ and $\left.\kappa_{s}\right)$ for $\mathrm{p}$ - and s-polarizations as follows,

$$
\begin{aligned}
& \kappa_{p}=\frac{\pi\left(n_{x} \cos ^{2} \theta_{\rho}-n_{z} \sin ^{2} \theta_{\rho}\right)}{\lambda \cos \theta_{\rho}} \\
& \kappa_{s}=\frac{\pi n_{y}}{\lambda \cos \theta_{\rho}}
\end{aligned}
$$

and $\xi=\Gamma d / 2 C_{\mathrm{s}}$ is expressed by following parameters:

$$
\begin{aligned}
& \Gamma=\Delta \theta K \sin \left(\varphi-\theta_{0}\right) \\
& C_{s}=\cos \left(\theta_{\rho}\right)-\left(\frac{K}{\beta}\right) \cos (\varphi)
\end{aligned}
$$

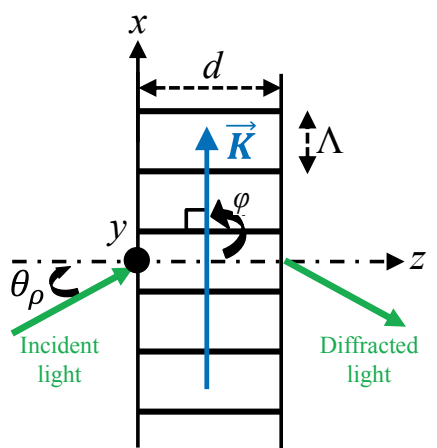

Fig. 1 Model of a thick grating.

The parameters described above are expressed as $\mathrm{K}=2 \pi / \Lambda$ and $\beta=2 \pi \mathrm{n}_{\mathrm{o}} / \lambda$. Here, $\mathrm{d}$ is the thickness of the grating, $\Delta \theta$ is the deviation between incidence angle $\left(\theta_{\rho}\right)$ and Bragg angle $\left(\theta_{0}\right)$ in the grating medium, $\varphi$ is the slanted angle, $n_{o}$ is the average refractive index, and $\Lambda$ is the grating spacing. In the present case, $\lambda$ and $d$ correspond to $0.532 \mu \mathrm{m}$ and $10 \mu \mathrm{m}$, respectively. The grating spacing $(\Lambda)$ is designed as $1 \mu \mathrm{m}$ and $0.25 \mu \mathrm{m}$ by adjusting the optical setup for laser interferometer. $n_{o}$ is estimated to be 1.532 from the mixture ratio of LC and polymer molecules. $\quad \mathrm{d}, \varphi$, and $\Lambda$ are confirmed by SEM observation, as detailed in later section. The amplitude of refractive-index modulation values of the present samples are estimated from the measurement of the incident angle dependence in anisotropic diffraction against the $\mathrm{p}$ - and s- polarizations using eq. (1). In HPDLC grating for an unslanted case $(\varphi=90), \mathrm{n}_{\mathrm{z}}$ is treated as same value with $\mathrm{n}_{\mathrm{y}}$ because the medium can be assumed to be optically uniaxial [8].

\section{EXPERIMENT}

\subsection{Sample preparation}

LC composite for an HPDLC grating was prepared using isotropic monomers (Kyoeisha Chemical) such as 2-hydroxy-3-phenoxy-propyl acrylate, 2-hydroxyethyl methacrylate, and dimethylol tricyclo decane diacrylate mixed with LC materials. The ratios of the isotropic monomers mixtures were 80,5 , and 15 wt. \%, respectively, and the nematic LC material (BL024, Merck) was added to the prepolymer mixture at content ratio of $25 \mathrm{wt} . \%$ based on the fraction of all ingredients. $\mathrm{N}$-phenylglycine and xanthene dye (dibromofluorescein) as a coinitiator and photoinitiator were introduced to the LC composites. The refractive index of the cured composition for isotropic monomers was found to be 1.520 by ellipsometric analysis. The physical properties such as extra ordinary and ordinary refractive indices $\left(\mathrm{n}_{\mathrm{e}}\right.$ and $\mathrm{n}_{\mathrm{o}}$ ) for BL024 material were supplied by Merck as 1.513 and 1.717. The glass cell used for the experiments had dimensions of $25 \mathrm{~mm} \times 20 \mathrm{~mm} \times 1 \mathrm{~mm}$, and it was assembled by combining a $10 \mu \mathrm{m}$ air gap spacer and two glass plates. The gap length corresponding to a film thickness of HPDLC sample was precisely measured by interferometry.

\subsection{Optical setup for device fabrication}

Figure 2 shows the optical setup to form the different grating spacing by varying the crossed angle of the laser beam. In Figs. 2(a) and (b), interferometric exposure using a green laser $\left(\mathrm{Nd}: \mathrm{YVO}_{4}, \lambda=532 \mathrm{~nm}\right)$ was adjusted to be crossed on the surface of the sample at 30 and 90 degrees. In Fig.2(b), the immersion technique with rectangular prisms was used to enter the laser beam at right angle on the surface of the sample. The interference pattern by laser exposure induces spatially periodic modulation in the refractive index at different grating spacing as $1 \mu \mathrm{m}$ and $0.25 \mu \mathrm{m}$ in the medium. The temperature for the device fabrication was adjusted to 50 ${ }^{\circ} \mathrm{C}$ using the temperature controller with the Peltier element in this setup. The laser source for photopolymerization was collimated and linearly polarized perpendicularly to the grating vector, and the intensity on the samples was fixed at $5 \mathrm{~mW} / \mathrm{cm}^{2}$. The polarization directions parallel and perpendicular to the grating vector are called p- and s-polarizations, respectively.

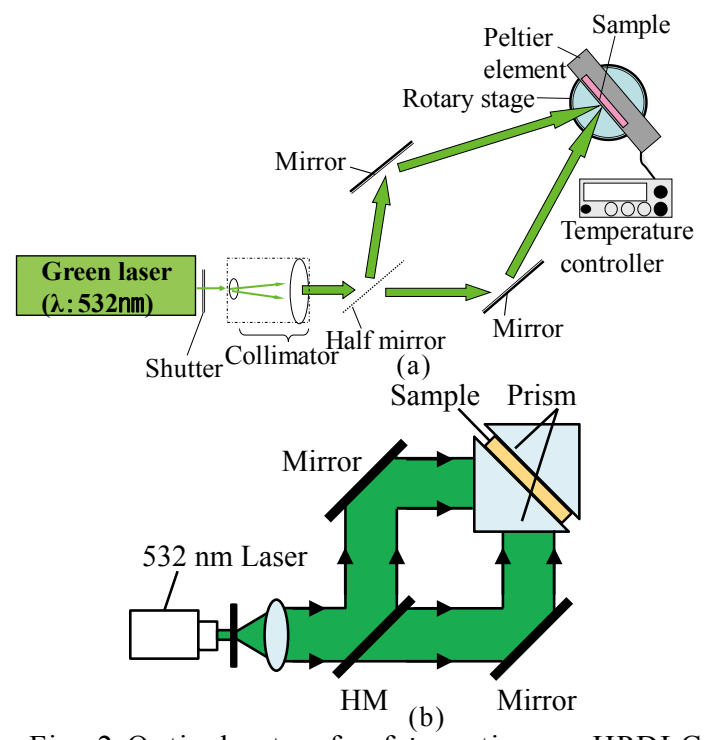

Fig. 2 Optical setup for fabricating an HPDLC grating at controlled temperature using laser interferometer.

\section{RESULTS AND DISCUSSION}

Figs. 3(a) and (b) show incidence angle dependences of diffraction efficiencies $\left(\eta_{p}\right.$ and $\left.\eta_{s}\right)$ for $p$ - and $s$ polarizations at unslanted HPDLC gratings $(\varphi=90)$. The square and circle marks correspond to grating spacing of 
$1 \mu \mathrm{m}$ and $0.25 \mu \mathrm{m}$. The effect of a film thickness on diffraction efficiencies of HPDLC gratings is estimated by parameter $\mathrm{Q}=2 \pi \lambda \mathrm{d} / \mathrm{n}_{0} \Lambda^{2}$ [14]. Diffraction conditions change from Raman Nath regime to Bragg regime with the increase of $\mathrm{Q}$ depending on the film thickness and grating spacing. The parameters $\mathrm{Q}$ are calculated as 21.8 and 349.1 for grating spacing of $1 \mu \mathrm{m}$ and $0.25 \mu \mathrm{m}$ by using those values shown in theoretical treatment section. Both gratings with $\mathrm{Q}>10$ can be treated as thick gratings defined in Bragg regime diffraction.

The solid lines show the theoretical predictions obtained using eq. (1). The diffraction efficiency was obtained as the ratio of diffracted first order intensity to the total of 0th and first order diffracted intensity. The theoretical predictions are well coincident with the experimental results. The diffraction efficiencies of $\eta_{\mathrm{p}}$ and $\eta_{\mathrm{s}}$ show $80 \%$ and $1.5 \%$ at grating spacing of $1 \mu \mathrm{m}$, while those of grating spacing of $0.25 \mu \mathrm{m}$ show $50 \%$ and $3 \%$, respectively. Though the diffraction efficiency for p-polarization decreases with the decrease of grating spacing, the obtained results show highly anisotropic diffraction properties since the diffraction efficiencies of $\eta_{\mathrm{p}}$ are much larger than $\eta_{\mathrm{s}}$ in both grating spacing. The resultant diffraction anisotropy, that is, the distinctive ratio $\left(\eta_{\mathrm{p}} / \eta_{\mathrm{s}}\right)$, is larger observed in the grating formed by the grating spacing of $1 \mu \mathrm{m}$ than that of $0.25 \mu \mathrm{m}$.
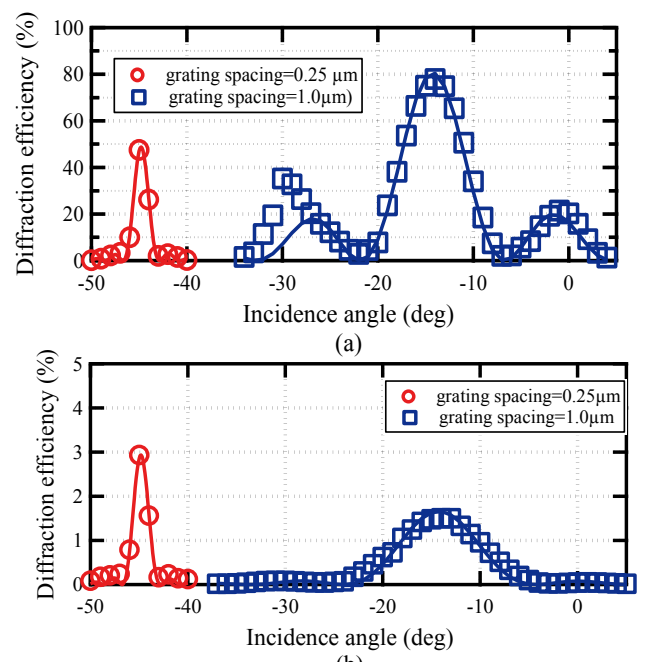

(b)

Fig. 3 Incidence angle dependences of diffraction in different grating spacing: (a) p- and (b) s-polarizations.

Figure 4 shows anisotropic refractive indices obtained based on the theoretical and experimental treatments in HPDLC gratings formed by the different grating spacing. The anisotropic refractive indices are obtained such as $\mathrm{n}_{\mathrm{x}}=0.038$ and $\mathrm{n}_{\mathrm{y}}=\mathrm{n}_{\mathrm{z}}=0.0025$ in the grating spacing of $1 \mu \mathrm{m}$, while they are $\mathrm{n}_{\mathrm{x}}=0.018$ and $\mathrm{n}_{\mathrm{y}}=\mathrm{n}_{\mathrm{z}}=0.002$ in the grating spacing of $0.25 \mu \mathrm{m}$. The anisotropic refractive index $\left(\mathrm{n}_{\mathrm{x}}\right)$ becomes larger than other refractive indices ( $n_{y}$ and $n_{z}$ ), and the ratio of $n_{x}$ to $n_{y}$ and $n_{z}$ indicates more or less than ten times at both gratings. The amplitude of $\mathrm{n}_{\mathrm{x}}$ corresponds to the direction of p-polarization which is parallel to the grating vector.

Figure 5 shows the SEM cross-sectional views of the HPDLC gratings formed by different grating spacing. Since the SEM observation of the grating with LC droplets is difficult, the LC molecules were rinsed out with methanol to observe the configuration of the LC droplet trace. Fig. 5(a) and (b) correspond to grating spacing of $1 \mu \mathrm{m}$ and $0.25 \mu \mathrm{m}$. The insets shown as A1 and $\mathrm{B} 1$ show the magnified images of cross-sectional shape. The close-up views show the solid and void regions corresponding to the cured polymer and the trace of LC droplets distributed to form a periodic structure. Though the region separated by the coalesced droplets in the LC layer of B1 is narrower observed compared with that of $\mathrm{A} 1$, the connectivity among the droplets is observed to exist in the LC layers formed at both grating spacing. The anisotropy in grating medium is considered to be affected by the orientations of LC molecules in LC phase and continuous coalesced droplets.

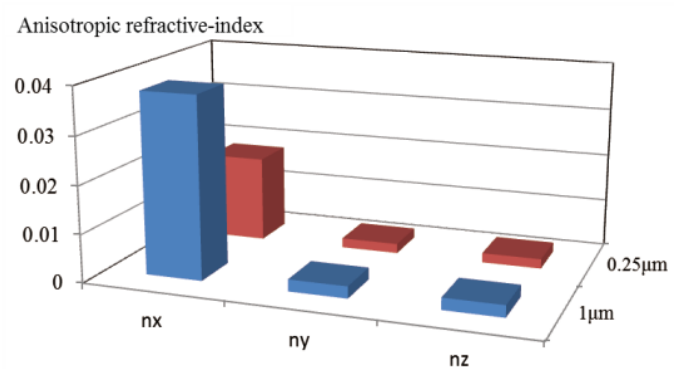

Fig. 4 Anisotropic refractive indices estimated in different grating spacing

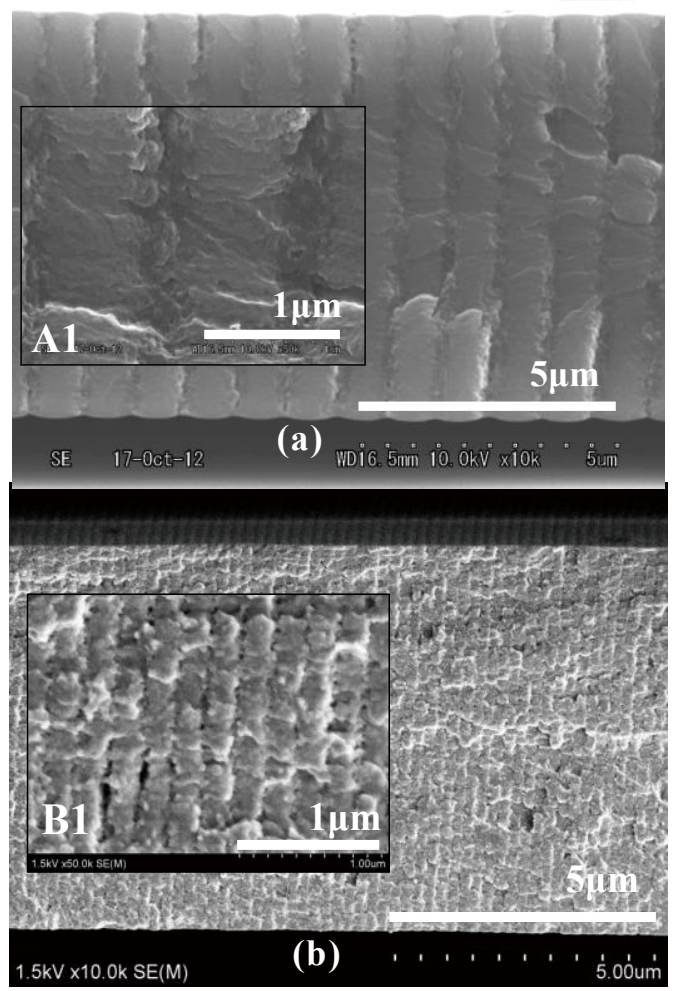

Fig. 5 SEM cross-sectional views of the HPDLC gratings formed by different grating spacing: (a) $1 \mu \mathrm{m}$ and (b) $0.25 \mu \mathrm{m}$. Insets shown as $\mathrm{A} 1$ and $\mathrm{B} 1$ are magnified views. 


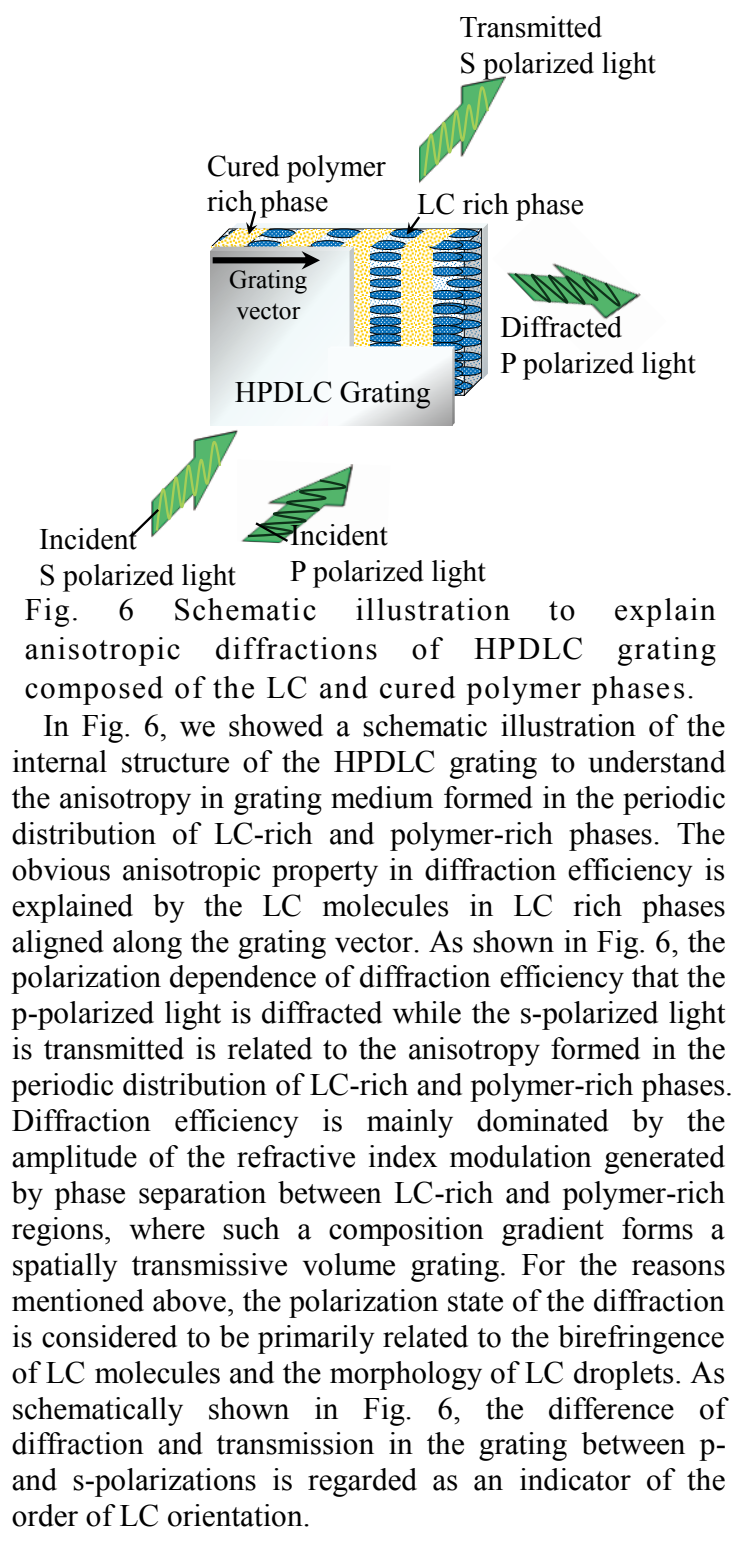

\section{CONCLUSIONS}

Anisotropic volume holographic gratings formed by the different grating spacing were produced from a polymer-dispersed liquid crystal. The fine grating structure was obtained by the laser interferometer using the immersion technique with rectangular prisms. The angular dependence of diffraction efficiencies were experimentally investigated for different grating structures. The theoretical predictions obtained by the equation based on the anisotropic diffractions were well coincident with the experimental results. The refractive-index modulations $\left(\mathrm{n}_{\mathrm{x}}, \mathrm{n}_{\mathrm{y}}, \mathrm{n}_{\mathrm{z}}\right)$ in grating medium corresponding to the three dimensional directions were estimated by applying theoretical calculation for the incidence angle dependence of the diffraction efficiencies. The anisotropic refractive index $\left(n_{x}\right)$ became larger than other refractive indices ( $n_{y}$ and $\mathrm{n}_{\mathrm{z}}$ ), and the ratio of $\mathrm{n}_{\mathrm{x}}$ to $\mathrm{n}_{\mathrm{y}}$ and $\mathrm{n}_{\mathrm{z}}$ indicated more or less than ten times in both gratings. The SEM observations showed the separated phase morphology of LC-rich and polymer-rich regions. The results demonstrated that the orientations of LC molecules in continuous coalesced droplets were susceptible to be aligned to the direction of p-polarization to be parallel along the grating vector.

\section{ACKNOWLEDGEMENT}

This work was supported in part by a JSPS Grant-in-Aid for Scientific Research C (26420328) and the Iwatani Naoji Foundation.

\section{REFERENCES}

[1] J. J. Butler, and M.S. Malcuit, "Diffraction properties of highly birefringent liquid-crystal composite gratings," Opt. Lett. 25, 420-422 (2000).

[2] T. J. Bunning, L.V. Natarajan, V.P. Tondiglia, and R. L. Sutherland, "Holographic polymer-dispersed liquid crystals (H-PDLCs)," Annu. Rev. Mater. Sci. 30, 83-115 (2000).

[3] C. C. Bowley, and G.P. Crawford, "Diffusion kinetics of formation of holographic polymer-dispersed liquid crystal display materials," Appl. Phys. Lett. 76, 2235-2237 (2000).

[4] R. L. Sutherland, V. P. Tondiglia, L.V. Natarajan, and T. J. Bunning, "Evolution of anisotropic reflection gratings formed in holographic polymer-dispersed liquid crystals," Appl. Phys. Lett. 79, 1420-1422 (2001).

[5] J. J. Butler, M. S. Malcuit, and M. A. Rodriguez, "Diffractive properties of highly birefringent volume gratings: investigation," J. Opt. Soc. Am. B 19, 183-189 (2002).

[6] R. L. Sutherland, "Polarization and switching properties of holographic polymer-dispersed liquid-crystal gratings. I. Theoretical model," J. Opt. Soc. Am. B 19, 2995-3003 (2002).

[7] M. E. Holmes, and M. S. Malcuit, "Controlling the anisotropy of holographic polymer-dispersed liquid-crystal gratings," Phys. Rev. E. 65, 066603-1-066603-4 (2002).

[8] I. D. Olenik, M. Fally, and M. A. Ellabban, "Temperature dependence of optical anisotropy of holographic polymer-dispersed liquid crystal transmission gratings," Phy. Rev. E. 74, 021707-1-1021707-10 (2006).

[9] A. Ogiwara, H. Kakiuchida, M. Tazawa, and H. Ono, "Analysis of anisotropic diffraction gratings using holographic polymer-dispersed liquid crystal," Jpn. J. Appl. Phys. 46, 7341-7346 (2007).

[10] A. Ogiwara, "Effects of anisotropic diffractions on holographic polymer-dispersed liquid-crystal gratings," Appl. Opt. 50, 594-603 (2011).

[11] A. Ogiwara, M. Watanabe, and R. Moriwaki, "Formation of temperature dependable holographic memory using holographic polymer-dispersed liquid crystal," Opt. Lett. 38, 1158-1160 (2013).

[12] A. Ogiwara, M. Watanabe, and R. Moriwaki, "Temperature dependence of anisotropic diffraction in holographic polymer-dispersed liquid crystal memory," Appl. Opt. 52, 6529-6536 (2013).

[13] H. Kogelnik, "Coupled wave theory for thick hologram gratings,” Bell Syst. Tech. 482909 -2947 (1969).

[14] M. G. Moharam, T. K. Gaylord, and R. Magnusson, "Criteria for Bragg regime diffraction by phase gratings," Opt. Commun. 32, 14-18 (1980).

(Received January 29, 2015; Accepted June 12, 2015) 